\title{
Prótesis Choostent como método de paliación de la disfagia secundaria a patología maligna*
}

\author{
Drs. MARIO ANSELMI M..$^{1,2}$, ANA MARÍA GEMMATO P. ${ }^{1,2}$, ANTONIO RODRÍGUEZ D. ${ }^{1,2}$
}

\author{
I Unidad Docente Asistencial de Cirugía Endoscópica. Hospital Regional de Concepción. \\ 2 Departamento de Cirugía Facultad de Medicina. Universidad de Concepción. \\ Concepción, Chile.
}

\begin{abstract}
Use of Choostent prosthetic device for palliation of dysphagia secondary to esophageal cancer
\end{abstract}

Background: Esophageal cancer causes disabling dysphagia and swallowing problems. Aim: To prospectively analyze the outcome of the insertion of a covered self-expanding metallic Choostent type prosthesis as a method of palliation of dysphagia, esophageal fistula or leak secondary to malignant disease of the esophagus or cardia. Material and Methods: A total of 30 consecutive patients aged $75 \pm 8.8$ years (63\% males) with malignant disease of the esophagus or cardia were studied. Results: In 27 patients (90\%) the stenosis was located in the esophagus, in two (6.7\%) at the gastroesophageal junction and in one (3.3\%) at the esophago - jejunal anastomosis due to tumor recurrence. In 24 cases $(80 \%)$, the indication of the prosthesis was dysphagia, in three $(10 \%)$ the presence of a tracheo-esophageal fistula and in the remaining three $(10 \%)$, the suspicion of a perforation. Patients were followed until death. The prosthesis was inserted without incidents in 29 patients $(96.6 \%)$. One patient had a pneumomediastinum, which evolved favorably. Dysphagia subsided in all patients. Early evolution was satisfactory in 12 patients $(66.7 \%)$. The most common early complication was a transient chest pain in 3 cases $(10 \%)$. Four patients $(13.3 \%)$ died within 30 days post procedure. Of the remaining 26 patients, $14(53.8 \%)$ had late complications (recurrence of dysphagia in $42 \%$ and hemorrhage in 11\%). The mean interval between stent insertion and death was $169 \pm 142.5$ days. Conclusions: The Choostent esophageal prosthesis improves dysphagia immediately and safely. However, its use is associated with a high rate of late complications, directly related to prosthetic dysfunction.

Key words: Esophageal cancer, dysphagia, self-expanding metal stents.

\section{Resumen}

Objetivo: Analizar en forma prospectiva los resultados de la inserción de una prótesis metálica autoexpandible cubierta tipo Choostent como método de paliación de la disfagia, fístulas o fugas esofágicas secundarias a patología maligna del esófago o cardias. Material y Método: Se estudiaron un total de 30 pacientes consecutivos portadores de patología maligna del esófago o de cardias. La edad promedio fue de $75 \pm 8,8$ años. $19(63,3 \%)$ eran del género masculino. En 27 pacientes (90\%) la estenosis comprometía el esófago, en 2

*Recibido el 9 de mayo de 2011 y aceptado para publicación el 28 de mayo de 2012.

Los autores no refieren conflictos de interés.

Correspondencia: Dr. Mario Anselmi M.

Casilla 160-C Fax: 56-41-2722581, Concepción, Chile.

manselmi@udec.cl 
$(6,7 \%)$ la unión gastroesofágica y en $1(3,3 \%)$ la anastomosis esófago yeyunal por recidiva tumoral. En 24/30 casos $(80 \%)$, la indicación de la prótesis fue la disfagia, en $3(10 \%)$ la presencia de fístula esófago-traqueal y en los 3 restantes (10\%), la sospecha de perforación. Los pacientes fueron controlados hasta su fallecimiento. Resultados: La prótesis se insertó sin incidentes en 29/30 pacientes (96,7\%). Un caso (3\%) presentó neumomediastino, que evolucionó favorablemente. La disfagia cedió en la totalidad de los enfermos. La evolución precoz fue satisfactoria en 12/30 pacientes (40\%), siendo la complicación precoz más frecuente el dolor torácico transitorio en 3 casos (10\%). Fallecieron precozmente 4 enfermos (13,3\%). De los 26 restantes, en 14 $(53,8 \%)$ se presentaron complicaciones tardías, siendo las más frecuentes la recidiva de la disfagia $(42,3 \%)$ y la hemorragia $(11,5 \%)$. Conclusión: Las prótesis Choostent mejoran la disfagia en forma inmediata y segura. Son efectivas en el manejo de las fístulas traqueo-esofágicas y perforaciones. Sin embargo, su uso se asocia a una alta tasa de morbilidad tardía directamente relacionada a la disfunción protésica.

Palabras clave: Cáncer de esófago, paliación de disfagia, prótesis metálicas autoexpandibles.

\section{Introducción}

Al momento del diagnóstico la mayoría de los enfermos con cáncer del esófago y del cardias presentan una enfermedad irresecable ${ }^{1,2}$. En Chile, la tasa de pacientes con esta condición fluctúa entre un $60 \%$ y un $84 \%^{3-5}$. En estos casos, la disfagia habitualmente constituye el principal y más molesto de los síntomas, por lo que la meta del tratamiento debe ser restaurar la habilidad de alimentarse y evitar las complicaciones derivadas de la estenosis neoplásica, como son la sialorrea, la aspiración y las fístulas. El método ideal de un tratamiento paliativo debería ser uno que solucione la disfagia rápidamente, en un solo intento y de forma permanente, que sea técnicamente simple y seguro de efectuar, bien tolerado por el paciente y que por lo tanto, la anestesia general no sea un requisito indispensable.

La terapia con prótesis autoexpandible metálica, cumple con la mayoría de las características de un tratamiento paliativo ideal ${ }^{6-10}$. Habitualmente es posible obtener un restablecimiento inmediato de la nutrición oral y una adecuada oclusión de fístulas traqueo-esofágicas ${ }^{11-13}$.

Aunque la inserción de una prótesis autoexpandible es un procedimiento seguro, no está exento de morbilidad. La tasa global de complicaciones intra o post procedimiento es de aproximadamente $6 \%{ }^{14-16}$. Las complicaciones tardías se presentan hasta en el $45 \%$ de los pacientes, siendo las más frecuentes la recidiva de la disfagia, la migración del stent, y la obstrucción del mismo ${ }^{14,17-19}$.

En la actualidad existen varios tipos de prótesis esofágicas autoexpandibles disponibles en el mercado. Todas tienen características de diseño con ventajas, como también desventajas específicas, pero sin diferencia significativa entre los diversos tipos estudiados ${ }^{20}$. No obstante, un estudio reciente encontró que el Niti-S stent cubierto tiene una mayor tasa de recidiva de la disfagia que el Niti-S stent de doble cubierta, posiblemente debido al aumento de la fuerza radial obtenido con este último ${ }^{21}$.
El objetivo del presente estudio es analizar en forma prospectiva los resultados de la inserción de una prótesis metálica autoexpandible cubierta tipo Choostent como método de paliación de la disfagia, fístulas o fugas esofágicas secundarias a patología maligna del esófago o cardias.

\section{Pacientes y Método}

Entre el 01 de febrero de 2007 hasta el 31 de agosto de 2008, se estudió en forma prospectiva, una serie consecutiva de 30 pacientes portadores de patología maligna del esófago y cardias que ingresaron a la Unidad Docente Asistencial de Cirugía Endoscópica (UDACE), del Hospital Regional de Concepción. Los enfermos recibieron tratamiento paliativo con prótesis esofágicas autoexpandibles metálicas tipo Choostent (MI Tech, Korea). Se seleccionaron aquellos casos con tumores menores de 12 $\mathrm{cm}$ de longitud, localizados a más de $2 \mathrm{~cm}$ distales al esfínter esofágico superior y con una sobrevida mayor a 4 meses. Esta última estimada por datos clínicos (World Health Organization Performance Status), según la presencia de metástasis y la longitud del tumor ${ }^{22}$. Se incluyeron además enfermos con fístula o perforación esofágica, aunque tuvieran una expectativa de vida menor. El estudio fue conducido siguiendo las normas de Buena Práctica Médica de acuerdo con las normas éticas vigentes (Declaración de Helsinki).

La edad promedio fue de $75 \pm 8,8$ años (rango: 61 a 93 años), 11 pacientes $(36,7 \%)$ eran del sexo femenino y los 19 restantes $(63,3 \%)$ del masculino. En 27 enfermos (90\%) la estenosis comprometía el esófago medio, en $2(6,7 \%)$ la unión gastroesofágica $y$ en $1(3,3 \%)$ la anastomosis esófago yeyunal por recidiva tumoral post gastrectomía total por cáncer. Lejos el tipo de neoplasia más frecuente fue el escamoso $(86,7 \%)$ El resto se muestra en la Tabla 1. La longitud promedio del tumor fue de $6,7 \pm 2,5 \mathrm{~cm}$ (Rango: 3 a $12 \mathrm{~cm}$ ), 19 pacientes $(63,3 \%)$ no habían 
Tabla 1. Tipo histológico del tumor

\begin{tabular}{|lcc|}
\hline & $\mathbf{n = 3 0}$ & $\mathbf{\%}$ \\
\hline Escamoso & 26 & 86,7 \\
\hline Adenocarcinoma & 3 & 10,0 \\
\hline Indiferenciado & 1 & 3,3 \\
\hline
\end{tabular}

recibido radioterapia previa a la inserción de la prótesis y en 11 casos (36,7\%), estas se instalaron como consecuencia de la recidiva tumoral post irradiación.

En 24 enfermos (80\%) la indicación de inserción de una prótesis autoexpandible fue la disfagia, en 3 pacientes $(10 \%)$, la fistulización del tumor hacia la vía aérea y en 3 casos (10\%), por sospecha o confirmación de perforación del esófago en el curso de una dilatación esofágica paliativa.

El grado de disfagia antes y después de la inserción fue evaluado según la clasificación de MellowPinkas $^{23}$ (Tabla 2). El índice promedio de disfagia pre-tratamiento fue de $3 \pm 0,69$. La mayoría de los pacientes toleraba sólo la ingesta de líquidos y 12 enfermos (40\%) estaban en afagia (Tabla 3 ).

La prótesis Choostent, consiste en una malla tubular autoexpandible hecha de una aleación de titanio y níquel (nitinol), completamente cubierta por una membrana de silicona. Está disponible en 4 medidas estándar según su longitud: 8, 11, 14 y 17 cm (Figura 1).

Todos los stents fueron insertados por un único cirujano endoscopista (M.A.M.). Previa anestesia tópica faríngea con lidocaína al 2\% y ubicación del enfermo en decúbito lateral izquierdo se procedió a efectuar la sedación mediante midazolam IV $(0,5-5$ $\mathrm{mg}$ ) y adicionando a algunos meperidina (10 a 30 $\mathrm{mg}$ ), dependiendo de la edad y de las condiciones generales de los pacientes.

La longitud de la lesión fue determinada por medio de radiología y endoscopia. En pacientes en los que no se pudo sobrepasar la estenosis con el endoscopio, previo a la inserción del stent, se dilató con bujías de Savary-Guillard (Wilson-Cook Medical Inc. Winston-Salem, N.C) hasta 38 Fr.

Los extremos proximal y distal de la lesión neoplásica fueron determinados mediante visión endoscópica directa y delimitados simultáneamente bajo fluoroscopia con marcadores externos radioopacos. Posteriormente, y conducido por una guía metálica de Savary, se insertó el stent hasta que sus extremos quedaran ubicados por lo menos $2 \mathrm{~cm}$ distal y proximal del tumor (Figura 2).

Una vez efectuada la liberación y comprobada la adecuada posición de la prótesis, se realizó un trán-
Tabla 2. Clasificación de la disfagia (Mellow-Pinkas)

\begin{tabular}{|cl|}
\hline Grado de disfagia & Síntomas \\
\hline 0 & Sin disfagia \\
\hline 1 & Tolera algunos sólidos \\
\hline 2 & Tolera papilla \\
\hline 3 & Ingiere sólo líquidos \\
\hline 4 & Afagia \\
\hline
\end{tabular}

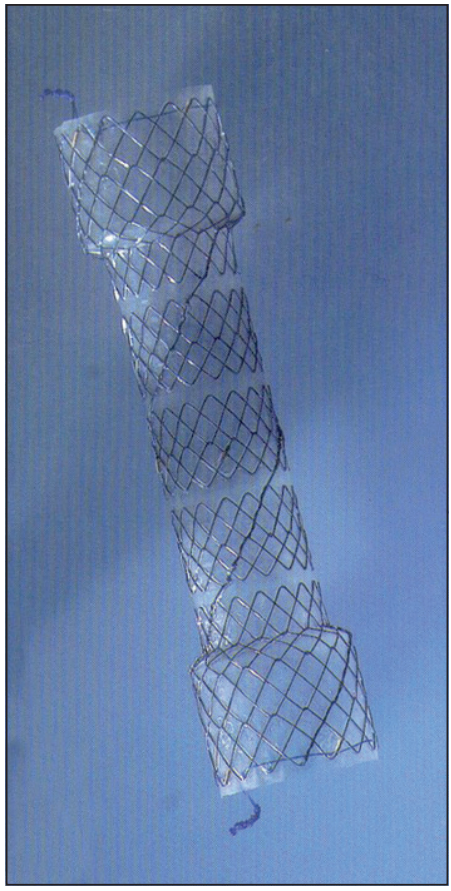

Figura 1. Prótesis Choostent.

sito esofágico con diatrizoato sódico (Hypaque ${ }^{\circledR}$ ) para descartar perforación o fuga. La posición del stent y su expansión se revisó a través de una nueva endoscopia y/o estudio radiológico, 48 horas tras su inserción (Figura 3).

A todos los pacientes se les instruyó verbalmente, con apoyo de un folleto estandarizado, de los cuidados y regímenes alimentarios que debían seguir tras la inserción de la prótesis. Asimismo, fueron citados mensualmente a control por consulta ambulatoria.

Se analizaron las complicaciones intraprocedimiento, precoces $(<30$ días) y tardías $(\geq 30$ días). El éxito clínico del tratamiento se definió como la mejoría del grado de disfagia con respecto al basal. El tiempo de seguimiento comprendió desde la inserción del stent hasta el fallecimiento del paciente. 


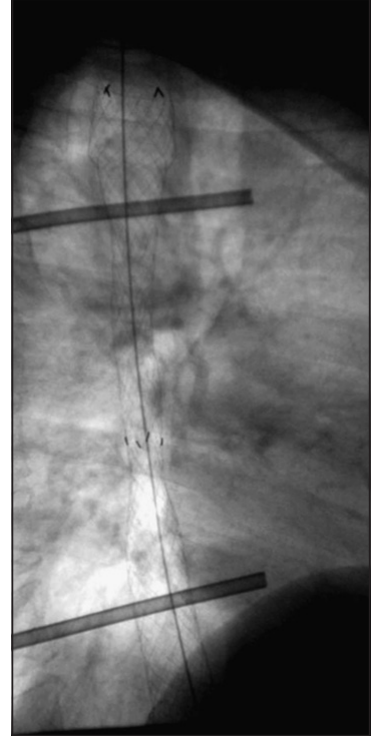

Figura 2. Inserción de protesis.

\section{Resultados}

Las prótesis fueron insertadas exitosamente en todos los casos, sin embargo, fue necesaria su reubicación, movilizándola hacia proximal en 8 pacientes $(26,7 \%)$ y hacia distal en $1(3,3 \%)$ traccionando el stent desde uno de los lazos destinados a esta maniobra (Figura 1).

Un paciente $(3,3 \%)$, portador de una neoplasia cardioesofágica, presentó dolor torácico inmediatamente posterior a la dilatación de la estenosis previa a la inserción de la prótesis. El estudio radiológico demostró la presencia de un enfisema mediastínico. Se procedió a insertar un stent cubierto con válvula antireflujo. El enfermo evolucionó con neumonía y sepsis por hongos, que respondió adecuadamente al tratamiento. El tránsito esofágico de control efectuado 72 horas más tarde, descartó filtración, por lo que se reinició la alimentación oral. El paciente fue dado de alta 2 semanas más tarde.

Se logró restaurar la ingesta oral en la totalidad de los enfermos, de estos 13 (43,3\%), toleraron una dieta normal (Tabla 3). El grado de disfagia promedio disminuyó desde $3,3 \pm 0,7$ previo a la paliación a $1,3 \pm 1,2$ posterior a la implantación de la prótesis $(\mathrm{p}<0,001)$.

La evolución precoz resultó satisfactoria en 20 de los 30 pacientes estudiados $(66,7 \%)$. Tres enfermos $(10 \%)$ presentaron dolor torácico transitorio, el que se trató con analgésico y cedió espontáneamente a las $72 \mathrm{hr}$ post inserción. Un paciente, con una lesión del esófago distal, debido a la inserción de una prótesis transcardial sin válvula, presentó reflujo

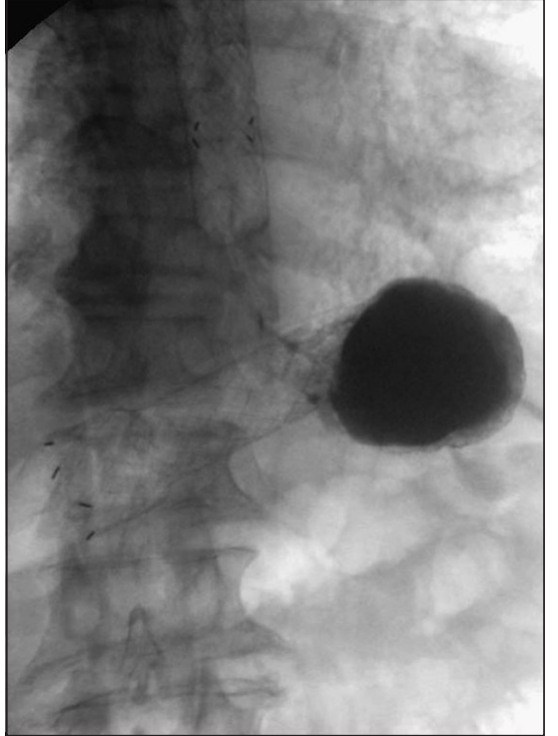

Figura 4. Prótesis fracturada.

gastroesofágico severo cuyos síntomas cedieron con medidas habituales. En una paciente en tratamiento con radioterapia, el control radiológico efectuado 20 días post inserción pesquisó fractura de la prótesis. Uno de los segmentos se encontraba en estómago y el otro in situ en el esófago (Figura 4). Ambos fragmentos fueron removidos endoscópicamente sin incidentes y se procedió a insertar un nuevo dispositivo. La paciente no presentaba disfagia al momento del retiro de la prótesis. El último enfermo, presentó fiebre que apareció $48 \mathrm{hr}$ post inserción y se mantuvo durante 5 días sin síntomas adicionales, no se logró precisar su causa, pero evolucionó satisfactoriamente y no hubo recidiva de esta complicación. (Tabla 4).

Del total, 4 casos $(13,3 \%)$ fallecieron de forma precoz $(8,9,24$ y 25 días post-inserción). Todos

Tabla 3. Grado de disfagia antes y después de la inserción de las prótesis

\begin{tabular}{|ccccc|}
\hline Grado disfagia & \multicolumn{2}{c}{$\begin{array}{c}\text { Pre- } \\
\text { procedimiento } \\
\text { n=30 }\end{array}$} & \multicolumn{2}{c|}{$\begin{array}{c}\text { Post- } \\
\text { procedimiento }\end{array}$} \\
n=30 & $\mathbf{\%}$ \\
0 & 0 & 0 & 13 & 43,3 \\
1 & 0 & 0 & 1 & 3,3 \\
\hline 2 & 4 & 13,3 & 11 & 35,7 \\
\hline 3 & 14 & 46,7 & 5 & 16,7 \\
4 & 12 & 40 & 0 & 0 \\
Promedio $\pm \mathrm{DE}$ & $3,3 \pm 0,77$ & $1,3 \pm 1,2 *$ \\
\hline
\end{tabular}

$* \mathrm{P}<0,001$. 
Tabla 4. Evolución precoz

\begin{tabular}{|lcc|}
\hline & $\mathbf{n}=\mathbf{3 0}$ & $\mathbf{\%}$ \\
\hline Satisfactoria & 20 & 66,7 \\
Dolor torácico transitorio & 3 & 10,0 \\
Reflujo gastroesofágico & 1 & 3,3 \\
Fractura de prótesis & 1 & 3,3 \\
Fiebre & 1 & 3,3 \\
\hline
\end{tabular}

Tabla 5. Evolución tardía

\begin{tabular}{|lcc|}
\hline & $\mathbf{n}=\mathbf{2 6}$ & $\mathbf{\%}$ \\
Satisfactoria & 12 & 46,2 \\
Recidiva de disfagia & 11 & 42,3 \\
Hemorragia digestiva alta & 3 & 11,5 \\
Migración de la prótesis & 1 & 3,8 \\
\hline
\end{tabular}

Tabla 6. Causas de recidiva de la disfagia

\begin{tabular}{|lcc|}
\hline Causa & $\mathbf{n = 1 1}$ & $\mathbf{\%}$ \\
\hline Fractura de prótesis & 4 & 36,4 \\
\hline Hiperplasia epitelial & 3 & 27,3 \\
Crecimiento tumoral & 2 & 18,2 \\
Migración de prótesis & 1 & 9,1 \\
\hline No determinada & 1 & 9,1 \\
\hline
\end{tabular}

se encontraban en afagia al momento de la instalación de la prótesis y uno de ellos presentaba una fístula esófagotraqueal. Dos enfermos evolucionaron con progresión del proceso infeccioso pulmonar que padecían previo a la inserción (neumonía por aspiración) y que fue finalmente la causa del deceso. El paciente con fístula esófago traqueal murió producto de una septicemia y el restante reingresó a los 25 días post inserción falleciendo de una falla multiorgánica de origen no precisado. La intubación esofágica, sin embargo, les permitió recuperar la alimentación por vía oral. Ningún paciente fue sometido a necropsia.

De los 26 pacientes que sobrevivieron más de un mes, en $14(53,8 \%)$ se presentaron 15 complicaciones tardías (Tabla $5)$. La reaparición tardía de la disfagia $(n=11)$ se debió en 4 casos $(36,4 \%)$, a la fractura total o parcial de la prótesis, en 3 de los cuales fue necesario insertar un segundo stent (Figuras 4 y 5). En 3 pacientes (11,5\%) ésta fue secundaria a una hiperplasia epitelial (Figura 6), la que fue solucionada con dilataciones en 2 de ellos y en el restante mediante la inserción de una segunda prótesis. En 2 enfermos (7,7\%) el crecimiento del tumor obstruyó total o parcialmente el extremo proximal del stent (Figura 7), uno de estos requirió

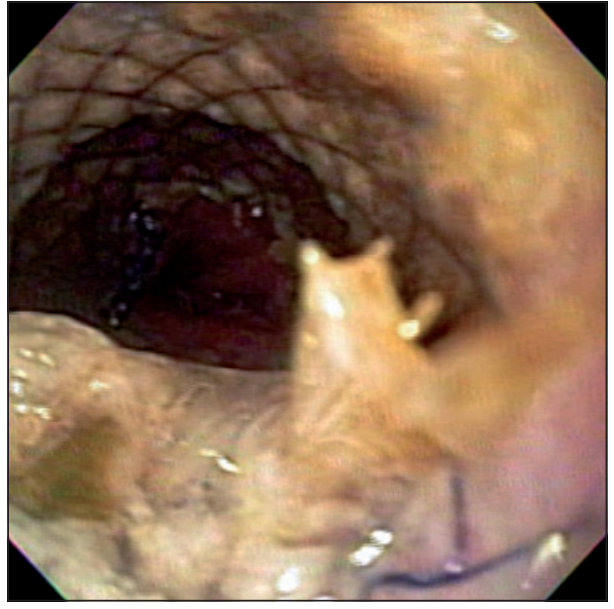

Figura 5. Fractura de prótesis. $2^{\circ}$ stent.

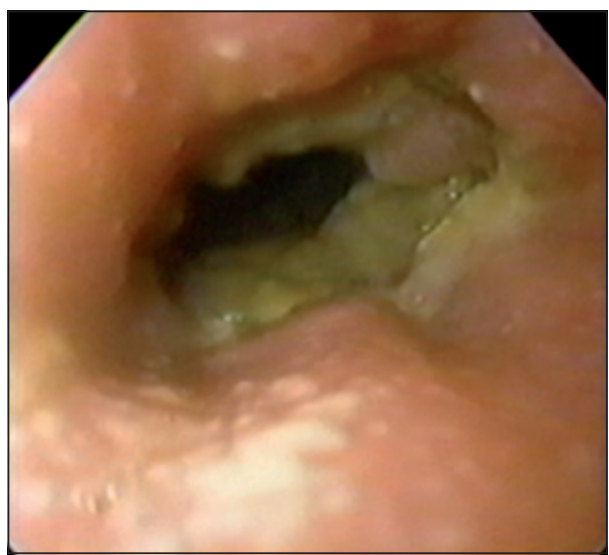

Figura 6. Hiperplasia epitelial ocasionando recidiva de la disfagia 6 meses post inserción.

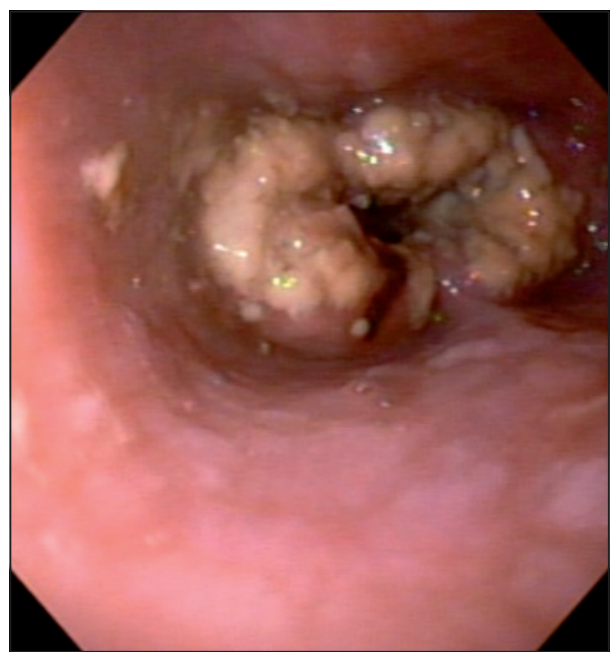

Figura 7. Sobrecrecimiento tumoral cubriendo el extremo proximal de la prótesis ocasionando recidiva tardía de la disfagia. 
de una segunda prótesis (Figura 8). Hubo 1 paciente $(3,8 \%)$ con migración de la prótesis hacia el estómago y 1 caso $(3,8 \%)$, en el que no se logró identificar una causa obstructiva que justificara la disfagia.

Se observaron 5 casos de fractura total o parcial de las prótesis $(16,7 \%)$, Todos estos stents fueron mayores o igual a los $140 \mathrm{~mm}$ de longitud. La neoplasia medía en promedio $7 \mathrm{~cm}$ y sólo un paciente, había recibido radioterapia. En 3 de estos enfermos se colocó una nueva prótesis, en uno se retiraron los fragmentos de la prótesis anterior y no se realizó ninguna otra medida terapéutica. Lo anterior, debido a que al momento del retiro de esta no había compromiso significativo de la nutrición oral y estaba programado el tratamiento de radioterapia en breve. El caso restante no fue tributario de terapéutica alguna, debido al deterioro avanzado de su estado clínico.

En los tres enfermos que presentaron como complicación tardía una hemorragia digestiva alta, esta fue masiva y con consecuencias fatales. Aunque no hubo necropsia, la causa de la complicación fue atribuida a las prótesis. La sobrevida promedio en esta serie fue de $169 \pm 142,5$ días (Rango: 8 y 647 días).

\section{Discusión}

Por muchos años, la paliación de la disfagia secundaria a una estenosis maligna irresecable del esófago fue llevada a cabo usando prótesis plásticas. En Chile, varios autores utilizaron este método, incluso con modelos hechos en casa ${ }^{24-26}$. La inserción de este tipo de elemento se asociaba a altas tasas de morbilidad y mortalidad ${ }^{24,26-28}$, especialmente en pacientes sometidos a tratamientos previos de radio y quimioterapia ${ }^{29}$.

Las prótesis metálicas autoexpandibles se han convertido en el tratamiento de elección para el manejo paliativo de la disfagia de origen maligno. El stent ideal debería presentar un amplio diámetro interno, ser cubierto, flexible, no traumático, de bajo grado de retracción, radiopaco, resistente a la migración, reubicable, removible y de bajo costo.

Las prótesis no cubiertas tienen bajas tasas de migración, pero el crecimiento tumoral dentro de ésta, puede ocurrir hasta en un 50\% de los $\operatorname{casos}^{30-33}$. Los stent cubiertos de nitinol, como el Choostent, ofrecen la ventaja de una alta flexibilidad y son potencialmente reubicables. Sus desventajas incluyen una baja radio-opacidad y una probable menor fuerza radial comparada con otras prótesis ${ }^{33}$. La inserción es fácil, simple y segura de efectuar. En efecto, en este estudio el éxito en la inserción del Choostent fue de un $100 \%$.

Otra ventaja de este tipo de stent es poseer lazos de recuperación en cada extremo, lo que las hace

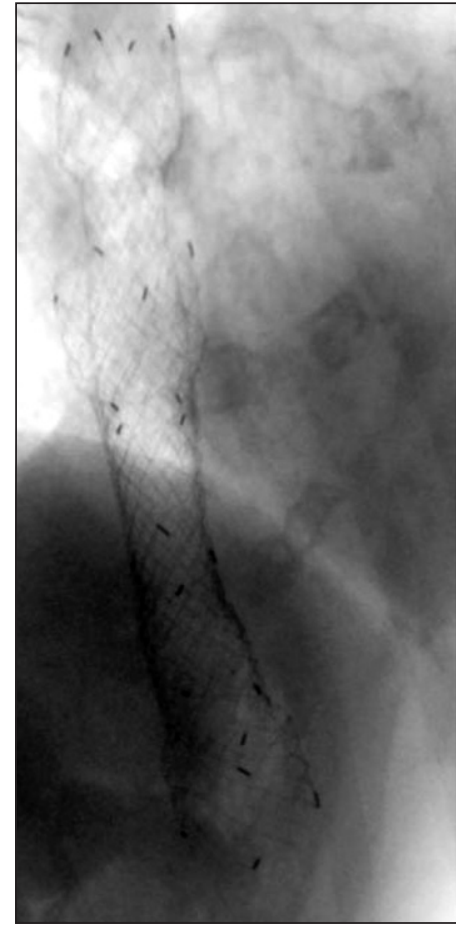

Figura 8. Inserción de prótesis adicional para solucionar la disfagia en un paciente con sobrecrecimiento tumoral.

reubicables e incluso removibles. De hecho, en este estudio una vez insertada la prótesis, el examen post procedimiento determinó que esta se encontraba en una posición no ideal en 9 de los 30 pacientes (30\%). La reubicación se hizo sin dificultad mediante la tracción de los lazos destinados a esta maniobra. La única complicación registrada durante el procedimiento fue secundaria a la dilatación previa y no a la inserción de la prótesis misma. Se obtuvo éxito clínico inicial en la totalidad de los pacientes.

Si se compara el desempeño inicial de la prótesis Choostent obtenido en esta serie, con los presentados por otros autores con diferentes tipos de stents, se observan resultados similares en el descenso del índice de disfagia ${ }^{6-10}$ (Tabla 7).

Los pacientes con fístula traqueo-esofágica presentan, por lo general, una sobrevida menor a un mes sin tratamiento específico ${ }^{34}$ y los intentos de cirugía paliativa se asocian a una alta mortalidad ${ }^{35}$. Es por eso que las prótesis metálicas autoexpandibles son el tratamiento de elección para la oclusión o bloqueo de las fístulas esófago-respiratorias ${ }^{34}$. En este estudio, las prótesis Choostent permitieron el reinicio de la dieta oral y el cese de los síntomas respiratorios derivados, en los tres pacientes con esta condición. Sin embargo, y a pesar de que hubo una mejoría significativa de la calidad de vida de estos enfermos, su sobrevida no superó a los 45 días en promedio. 
Tabla 7. Éxito clínico con el uso de prótesis metálicas autoexpandibles (Basada en la escala de Mellow-Pinkas pre y post inserción)

\begin{tabular}{|c|c|c|c|c|}
\hline Autor & $\mathbf{n}$ & Prótesis & $\begin{array}{c}\text { Disfagia } \\
\text { pre inserción }\end{array}$ & $\begin{array}{c}\text { Disfagia } \\
\text { post inserción }\end{array}$ \\
\hline Bona $^{10}$ & 32 & Choostent & 3,5 & 2,1 \\
\hline Bona $^{10}$ & 33 & Ultraflex & 3,4 & 1,9 \\
\hline Van Boeckel ${ }^{8}$ & 37 & Wallflex & 3 & 1 \\
\hline Verschuur $^{6}$ & 42 & Niti-S stent & 3 & 0 \\
\hline Van Boeckel ${ }^{7}$ & 44 & Evolution & 3 & 1 \\
\hline Uitdehaag ${ }^{9}$ & 45 & Alimaxx-E & 3 & 1 \\
\hline UDACE & 30 & Choostent & 3,2 & 1,3 \\
\hline
\end{tabular}

Tabla 8. Complicaciones con el uso de prótesis metálicas autoexpandibles

\begin{tabular}{|lcccc|}
\hline Autor & n & Hemorragia & Migración & $\begin{array}{c}\text { Recurrencia } \\
\text { disfagia }\end{array}$ \\
\hline Bona $^{10 \S}$ & 32 & - & $3,2 \%$ & $17 \%$ \\
Bona $^{10 ¥}$ & 33 & - & $6,1 \%$ & $9 \%$ \\
\hline Van Boeckel $^{8}$ & 37 & - & $8,1 \%$ & $21,6 \%$ \\
Verschuur $^{6}$ & 42 & $4,8 \%$ & $36 \%$ & $11,9 \%$ \\
Van Boeckel $^{7}$ & 44 & $2,3 \%$ & $5 \%$ & $9 \%$ \\
\hline Uitdehaag $^{9}$ & 45 & $8,8, \%$ & $35,5 \%$ & $49 \%$ \\
\hline UDACE & 30 & $10 \%$ & $6,7 \%$ & $36,7 \%$ \\
\hline
\end{tabular}

${ }^{\S}$ Choostent. ${ }^{¥}$ Ultraflex.

La mortalidad a los 30 días en esta serie fue de 13,3\% (4/30 pacientes). Todas secundarias a una evolución desfavorable de cuadros infecciosos previos o de enfermedad avanzada (fístula traqueo-esofágica).

El stent cubierto previene el crecimiento del tumor pero está asociado con una tasa más alta de migración ${ }^{38}$, siendo en el presente estudio del orden del $6,7 \%$, cifra comparable a la reportada por otros autores $\mathrm{s}^{6-10}$ (Tabla 8 ). Habitualmente la prótesis migrada queda retenida en el estómago y generalmente no se asocia a complicaciones, siendo la progresión hacia segmentos más distales un evento poco frecuente ${ }^{37}$. En esta casuística, los pacientes que presentaron esta complicación, fueron sometidos a extracción del stent o reubicación endoscópica del mismo, sin incidentes. Una ventaja significativa del diseño de la prótesis Choostent, si ocurre este problema, es la presencia de lazos en los extremos proximal y distal que facilitan su movilización y potencialmente su extracción ${ }^{10}$.

En esta serie, la recidiva de la disfagia se presentó en 11 pacientes $(36,7 \%)$. Esta cifra es alta, aunque dentro de los límites reportados en la literatura utilizando otro tipo de stent. Esta complicación se presentó en promedio a los 127 días (Rango: 40-270 días). La principal causa de reaparición o empeoramiento de la disfagia fue la disfunción de la prótesis, (fractura y migración). Si se excluye esta causa, la tasa de recidiva de la disfagia desciende a sólo un $20 \%$, cifra similar a la de otros autores ${ }^{6-10}$.

La tasa de fractura de la prótesis Choostent para este estudio fue de $16,7 \%$. Esta complicación es prácticamente inexistente en estudios similares con otros tipos de próte$\operatorname{sis}^{6-10}$. Como posibles causas que predisponen a la rotura del stent, se describen la combinación de debilidad intrínseca de la propia prótesis y factores corrosivos no totalmente identificados ${ }^{37}$. En el presente trabajo, como característica común, se encontró que esta complicación se presentó en prótesis de longitud mayor o igual a los $140 \mathrm{~mm}$.

La mayoría de las complicaciones fueron manejadas endoscópicamente, pero añadieron costos significativos al tratamiento. Algunas de ellas, como la hemorragia, tienen una alta tasa de morbimortalidad. En esta serie se presentaron 3 casos de hemorragia digestiva alta $(10 \%)$, todas con consecuencias fatales y atribuidas a la prótesis, aunque no se realizaron autopsias para confirmar su asociación. Esta tasa es levemente superior a las reportadas por otros autores ${ }^{6-10}$, probablemente debido a que en el presente estudio se efectuó un seguimiento más prolongado. No obstante, el sangrado digestivo se presenta hasta en un $8 \%$ de los pacientes con cáncer esofágico como evolución natural de la enfermedad, por lo que factores independientes a la prótesis pueden jugar un rol importante en esta complicación ${ }^{12,38,39}$.

De los resultados de este trabajo se concluye que las prótesis esofágicas Choostent mejoran la disfagia secundaria a patología maligna en forma inmediata, segura y prolongada. Son efectivas en el manejo de las fístulas traqueo-esofágicas y las perforaciones producidas en el curso de una dilatación endoscópica paliativa. Sin embargo, su uso se asocia a una alta tasa de morbilidad tardía directamente relacionada a la disfunción protésica. 


\section{Referencias}

1. Parkin DM, Bray F, Ferlay J, Pisani P. Global cancer statistics 2002. CA Cancer J Clin. 2005;55:74-108.

2. Siersema PD. Esophageal cancer. Gastroenterol Clin North Am. 2008;37:943-64.

3. González F, Escobar I, Garrido R, Gatica A. Experiencia en cirugía resectiva del cáncer esofágico Rev Chil Cir 1997;49:483-7.

4. Venturelli A, Soto S, Díaz J, Cardemil B, Sánchez A, Jiménez L. Cáncer de esófago, tratamiento en el Hospital Clínico Regional de Valdivia durante el período 1982 -2001. Rev Chil Cir. 2003;55:381-4.

5. Csendes A, González G. Cirugías digestivas más frecuentes en Chile, excluyendo colorectal. Rev Chil Cir. 2008;60:379-86.

6. Verschuur EM, Homs MY, Steyerberg EW, Haringsma J, Wahab PJ, Kuipers EJ, et al. A new esophageal stent design (Niti-S stent) for the prevention of migration: a prospective study in 42 patients. Gastrointest Endosc. 2006;63:134-40.

7. van Boeckel PG, Siersema PD, Sturgess R, Dwyer L, Raijman I, Hirdes MM, et al. A new partially covered metal stent for palliation of malignant dysphagia: a prospective follow-up study. Gastrointest Endosc. 2010; 72:1269-73.

8. van Boeckel PG, Repici A, Vleggaar FP, Solito B, Rando G, Cortelezzi C, et al. A new metal stent with a controlled-release system for palliation of malignant dysphagia: a prospective, multicenter study. Gastrointest Endosc. 2010;71:455-560.

9. Uitdehaag MJ, van Hooft JE, Verschuur EM, Repici A, Steyerberg EW, Fockens P, et al. A fully-covered stent (Alimaxx-E) for the palliation of malignant dysphagia: a prospective follow-up study. Gastrointest Endosc. 2009;70:1082-9.

10. Bona D, Laface L, Bonavina L, Abate E, Schaffer M, Ugenti I, et al. Covered nitinol stents for the treatment of esophageal strictures and leaks. World J Gastroenterol. 2010;16:2260-4.

11. Saxon RR, Morrison KE, Lakin PC, Petersen BD, Barton RE, Katon RM, et al. Malignant esophageal obstruction and esophagorespiratory fistula: palliation with a polyethylene-covered Z-stent. Radiology 1997;202:349-54.

12. Ell C, May A, Hahn EG, Gianturco-Z. Stents in the palliative treatment of malignant esophageal obstruction and esophagotracheal fistulas. Endoscopy 1995;27:495500 .

13. Morgan R, Ellul J, Denton E, Glynos M, Mason R, Adam A. Malignant esophageal fistulas and perforations: management with plastic-covered metallic endoprostheses. Radiology 1997;204:527-32.

14. Baron TH. Expandable metal stents for the treatment of cancerous obstruction of the gastrointestinal tract. N Engl J Med. 2001;344:1681-7.
15. Baron TH. Minimizing endoscopic complications: endoluminal stents. Gastrointest Endosc Clin N Am. 2007; 17:83-104.

16. Ramirez FC, Dennert B, Zierer ST, Sanowski RA. Esophageal self-expandable metallic stents--indications, practice, techniques, and complications: results of a national survey. Gastrointest Endosc. 1997;45:360-4.

17. Hendra KP, Saukkonen JJ. Erosion of the right mainstem bronchus by an esophageal stent. Chest. 1996;110:857-8.

18. Homs MY, Siersema PD. Stents in GI tract. Expert Rev Med Devices 2007; 4 :741-52.

19. Javle M, Ailawadhi S, Yang GY, Nwogu CE, Schiff MD, Nava HR. Palliation of malignant dysphagia in esophageal cancer: a literature-based review. J Support Oncol. 2006;4:365-73 .

20. Sreedharan A, Harris K, Crellin A, Forman D, Everett SM. Interventions for dysphagia in oesophageal cancer. Cochrane Database System Rev. 2009.

21. Battersby NJ, Bonney GK, Subar D, Talbot L, Decadt B, Lynch N. Outcomes following oesophageal stent insertion for palliation of malignant strictures: A large single centre series. J Surg Oncol. 2012;105:60-5.

22. Steyerberg EW, Homs MY, Stokvis A, Essink-Bot ML, Siersema PD. Stent placement or brachytherapy for palliation of dysphagia from esophageal cancer: a prognostic model to guide treatment selection. Gastrointest Endosc. 2005;62:333-40.

23. Mellow MH, Pinkas H. Endoscopic laser therapy for malignancies affecting the esophagus and gastroesophageal junction. Analysis of technical and functional efficacy. Arch Intern Med. 1985;145:1443-6.

24. Braghetto I, Rebolledo P, Csendes A, Braghetto H. Confección y colocación de prótesis endoluminales para cáncer de esófago y cardias. Rev Chil Cir. 1990;42:204.

25. Kirberg A, Morales X, Brito J, Mordo P, Branimir S, Prótesis esofágica: inserción endoscópica. Experiencia preliminar. Rev Chil Cir. 1991:43:354-7.

26. Braghetto I, Amat P, Rebolledo P, Ferrario M . Tratamiento paliativo del cáncer de esófago irresecable. Rev Chil Cir. 1994;46 :377-384.

27. Gasparri G, Casalegno P, Camandona M, Dei Poli M, Salizzoni M, Ferrarotti G. et al. Endoscopic insertion of 248 prostheses in inoperable carcinoma of the esophagus and cardia: Short terms and long terms results. Gastrointest Endosc. 1987;33:354-6.

28. Tygat G. Endoscopic therapy of the esophageal cancer: possibilities and limitations. Endoscopy 1990;22:263-7.

29. Kinsman K, DeGregorio B, Katon R. Prior radiation and chemotherapy increase the risk of life threatening complications after insertion of metallic stents for esofagogastric malignancy. Gastrointest Endosc.1996; 43:1996-203.

30. Rodríguez J, Porse G, Gerlach U, Manegold B. Tratamiento paliativo de las estenosis tumorales inoperables 
del esófago y estómago con prótesis metálicas autoexpandibles Rev Chil Cir. 1995;47:444-8.

31. Saranovic D, Djuric-Stefanovic A, Ivanovic A, Masulovic D, Pesko P. Fluoroscopically guided insertion of self-expandable metal esophageal stents for palliative treatment of patients with malignant stenosis of esophagus and cardia: comparison of uncovered and covered stent types. Dis Esophagus 2005;18:230-8.

32. Vakil N, Morris AI, Marcon N, Segalin A, Peracchia A, Bethge $\mathrm{N}$, et al. A prospective, randomized, controlled trial of covered expandable metal stents in the palliation of malignant esophageal obstruction at the gastroesophageal junction. Am J Gastroenterol. 2001;96:17916.

33. van Boeckel PG, Repici A, Vleggaar FP, Solito B, Rando G, Cortelezzi C, et al. A new metal stent with a controlled-release system for palliation of malignant dysphagia: a prospective, multicenter study. Gastrointest Endosc. 2010;71:455-560.

34. Choi MK, Park YH, Hong JY, Park HC, Ahn YC, Kim $\mathrm{K}$, et al. Clinical implications of esophagorespiratory fistulae in patients with esophageal squamous cell car- cinoma (SCCA). Med Oncol. 2010;27:1234-8.

35. Conlan AA, Nicolaou N, Delikaris PG, Pool R. Pessimism concerning palliative bypass procedures for established malignant esophagorespiratory fistulas: a report of 18 patients. Ann Thorac Surg. 1984;37:10810.

36. Vakil N, Morris A, Marcon N, Segalin A, Peracchia A, Bethge $\mathrm{N}$, et al. A prospective, randomized, controlled trial of covered expandable metal stents in the palliation of malignant esophageal obstruction at the gastroesophageal junction. Am J Gastroenterol. 2001;96:17916.

37. Reddy AV, Alwair H, Trewby PN. Fractured esophageal nitinol stent: Report of two fractures in the same patient. Gastrointest Endosc. 2003;57:138-9.

38. Randall J, Obeid ML, Blackledge GR. Haemorrhage and perforation of gastrointestinal neoplasms during chemotherapy. Ann R Coll Surg Engl. 1986;68:286-9.

39. Savides TJ, Jensen DM, Cohen J, Randall GM, Kovacs TO, Pelayo E, et al. Severe upper gastrointestinal tumor bleeding: endoscopic findings, treatment, and outcome. Endoscopy 1996;28:244-8. 